\title{
Reflets
}

Revue ontaroise d'intervention sociale et communautaire

\section{Le cours d'été en promotion de la santé, session 1999}

\section{Colette Fraser}

Volume 5, numéro 2, automne 1999

La santé des francophones de l’Ontario

URI : https://id.erudit.org/iderudit/026287ar

DOI : https://doi.org/10.7202/026287ar

Aller au sommaire du numéro

Éditeur(s)

Reflets : Revue ontaroise d'intervention sociale et communautaire

ISSN

1203-4576 (imprimé)

1712-8498 (numérique)

Découvrir la revue

Citer cet article

Fraser, C. (1999). Le cours d'été en promotion de la santé, session 1999. Reflets, 5(2), 270-278. https://doi.org/10.7202/026287ar

Tous droits réservés (C) Reflets : Revue ontaroise d'intervention sociale et communautaire, 1999
Ce document est protégé par la loi sur le droit d'auteur. L'utilisation des services d’Érudit (y compris la reproduction) est assujettie à sa politique d'utilisation que vous pouvez consulter en ligne.

https://apropos.erudit.org/fr/usagers/politique-dutilisation/ 


\section{Le cours d'été en promotion de la santé, session 1999}

\section{Colette $F$ raser}

Travailleuse autonome, coordonnatrice du cours d'été en promotion de la santé, session 1999

\section{Introduction}

Depuis longtemps, on parle de l'importance de former les formatrices et les formateurs dans le domaine de la santé. $0 \mathrm{r}$, le Cours d'été en promotion de la santé (CÉPS) cherche justement à répondre à ce besoin depuis bientôt six ans dans la province de I'O ntario. Le ministère de la Santé de l'O ntario et le C entre for $\mathrm{H}$ ealth Promotion de I'U niversité de Toronto parrainent ce projet depuis ses débuts. Pourtant, l'étendue de cette expérience éducative dépasse largement le cadre et l'enceinte de l'institution universitaire. C ela s'est avéré particulièrement vrai lors de l'édition 1999 du cours d'été qui a lieu à l'U niversité Laurentienne de Sudbury du 31 mai au 3 juin. En plus d'avoir lieu dans le $\mathrm{N}$ ord de l'O ntario pour la première fois, ce cours d'été comportait trois volets culturels, soit les volets anglophone, francophone et autochtone. L'ajout d'une composante autochtone, I'emphase sur le partage, la culture, la créativité, l'avenir et la technologie ont fait de l'édition 1999 un moment inoubliable. Par exemple, on retiendra le silence absolu de l'auditoire devant les sonsenvoûtant des tambours et des chants autochtones retentissant dans l'auditorium Fraser ou l'enthousiasme des participants créant des images selon leurs couleurs intérieures, sur des bannières 
accrochées dans les corridors de l'édifice des classes. C et article raconte comment le CÉPS a été offert dansla région de Sudbury, la nouveauté de l'expérience nord ontarienne et la nécessité de maintenir le caractère itinérant du cours pour répondre aux besoins des intervenantes et des intervenants en santé de l'O ntario.

\section{Un aperçu du cours d'été en promotion de la santé}

\section{Historique}

Les principaux promoteurs du cours ont été le ministère ontarien de la Santé, le $\mathrm{C}$ entre for $\mathrm{H}$ ealth P romotion de I'U niversité deToronto, le $C$ entre ontarien d'intervention et de prévention, les services de santé publique, les centres de santé communautaires et plusieurs autres organismes œuvrant dans divers secteurs attachés à la santé. Le CÉPS est offert annuellement depuis 1994. De 1994 à 1996, le cours s'est tenu àToronto, puis, en 1997, à 0 ttawa. En 1998, le cours s'est déroulé à nouveau dans la métropole ontarienne. Le cours dure généralement de deux à quatre jours. Le CÉPS répond aux besoins de ceux et celles qui ont peu de connaissances en promotion de la santé comme à ceux et celles qui ont déjà des notions de base et possèdent de l'expérience dans le domaine.

\section{Objectifs}

L'objectif général du CÉPS est de permettre aux praticiennes et aux praticiens de la santé d'acquérir des connaissances et des habiletés nouvelles qui leur permettront de planifier, de mettre en œuvre et d'évaluer des stratégies de promotion de la santé dans leur milieu respectif. C ette année, le cours cherchait plus particulièrement à donner l'occasion aux participantes et aux participants:

1. d'accroître leurs connaissances et leurs habiletés en matière de promotion de la santé en vue d'améliorer leurs communautés;

2. d'établir des contacts utiles avec les autres participants; 
3. de partager les différences culturelles dans le domaine de la promotion de la santé et,

4. d'apprendre de nouvelles façons d'utiliser la technologie dans le domaine de la promotion de la santé.

\section{Clientèle}

Le CÉPS cible lesmembres de groupes communautai res, débutants ou chevronnés, dans le domaine de la promotion de la santé. Les participantes et participants proviennent de différents milieux comme les centres de santé communautaire, les bureaux de santé, les écoles, les instances gouvernementales. C ette année, le CÉPS a attiré une nouvelle clientèle de la région du $\mathrm{N}$ ord de la province qui, antérieurement, ne pouvait pas se déplacer versToronto ou ne bénéficiait pas des sommes nécessaires à un tel déplacement.

\section{Planification et coordination}

La coordonnatrice, secondée par un comité planificateur comprenant trois sous- comités ont assuré la planification des trois volets du CÉPS, édition 1999. Le mandat des trois sous-comités est respectivement d'offrir des conseils et de participer à l'élaboration et la mise en œuvre : 1) du curriculum, 2) du plan promotionnel et, 3) du plan d'évaluation.Au sein de ces comités, on a veillé à assurer la représentation de divers types d'organismes: bureaux de santé, centres de santé communautaire, agences gouvernementales, organismes communautaires francophones, conseils de santé, etc.

\section{Structure}

Le CÉPS 1999 a adopté la même structure que celles des années passées, tout en privilégiant le contenu portant sur les valeurs culturelles des diverses composantes. À cette fin, et pour encourager l'intégration et l'échange culturel, un chevauchement étroit entre les trois volets a été établi, tout en respectant les besoins des intervenants œuvrant dans les milieux anglophone, francophone et autochtone. Les thèmes de base ayant servi à l'élaboration des ateliers et des activités du CÉPS furent le partage, la culture, la technologie, les services télématiques, la créativité et l'avenir. 


\section{Types de sessions}

\section{Sessions plénières mixtes}

U ne session ayant pour thème L'équilibre dans la promotion de la santé a réuni les participantes et les participants des diverses composantes autour de la nécessité d'intégrer les quatre aspects de la personne dans le domaine de la promotion de la santé. U ne autre session, offerte par vidéoconférence, portant sur L'A venir en promotion de la santé dans une perspective globale a permis d'unir six représentants de l'U nion internationale de promotion de la santé et d'éducation pour la santé, qui se rencontraient à Q uébec, aux participantes et participants du CÉPS à Sudbury. Enfin, la session plénière de clôture ayant pour titre $D$ reamweavers, dreammakers, incitait les pourvoyeurs de services à poursuivre leur rêve en promotion de la santé dans leur communauté respective, malgré les coupures budgétaires ou salariales et les contretemps qui secouent le domaine.

Par ailleurs, pour exaucer un vœu formulé lors du dernier CÉPS, une soirée sociale a été organisée afin de favoriser la création de liens et la formation de réseaux entre les diverses composantes. C ette soirée aux couleurs de la francophonie, avec son souper et son spectacle de chansons d'É dith Piaf, a vraiment transformé la Caverne de Science- $\mathrm{N}$ ord, en un grand bistrot parisien.

\section{Ateliers parallèles}

Les ateliers parallèles durent six heures et permettent à chacune des composantes d'aborder un sujet en profondeur. C haque atelier comprend un exposé théorique et des formations pratiques que les participantes et les participants peuvent utiliser lorsqu'elles ou ils retournent dans leur communauté respective. Par exemple, un des ateliers de la composante anglophone avait pour titre: 0 utcome E valuation and Poverty: Strategies for Sodal A cion. D ansla composante autochtone, les ateliers cherchaient soit à répondre à des questions comme $\mathrm{H}$ owW estern andT raditional $\mathrm{H}$ ealth $\mathrm{C}$ are $\mathrm{C}$ an W ork $\mathrm{C}$ reatively Together? ou d'examiner un passé encore douloureux comme 
$\mathrm{H}$ ealing the Past, $\mathrm{C}$ reating the $\mathrm{F}$ uture: the Impad of $\mathrm{R}$ esidential $\mathrm{S}$ chools U n des ateliers de la composante francophone présentait les données récentes sur la santé de cette population en O ntario, données recueillies lors de l'Enquête sur la santé en O ntario 19961997 et du R ecensement 1996. C et atelier avait pour thème: Les $F$ rancophones, nos besoins, notre santé - des pratiques à notre image. $U \mathrm{n}$ autre atelier, intitulé $D$ es stratégies de développement communautaire: outils pratiques pour les intervenants communautaires faisait le profil d'un projet communautaire et offrait les moyens pour débuter un programme semblable. Enfin, un troisième atelier ayant pour thème $L$ es programmes de promotion de la santé en milieu de travail C omment vendre sa salade? présentait les enjeux, les bienfaits et les difficultés d'intégrer ces programmes dans le milieu de travail.

\section{Boîte à outils}

À l'intérieur de chacune des composantes, les participantes et participants devaient choisir parmi dix ateliers d'une durée approximative de 90 minutes consacrées à l'étude d'une myriade de démarches de santé qui ont cours dans la province. C es ateliers, appelées aussi «boîtes à outils», abordaient des expériences aussi diverses que L es T hérapies complémentaires, L e T héâtre du Soleil couchant, Le défi de la promotion de la santé dans les centres de santé en milieux ruraux et du $\mathrm{N}$ ord, $\mathrm{T}$ he $\mathrm{C}$ hallenge ofW orking with $\mathrm{L}$ ow Income W omen, T he M edicineW heel, etc

\section{Expérience unique de 1999}

Comme l'a dit Francine Deroche, consultante pour la firme Innovaction et responsable de la synthèse de clôture, l'édition 1999 du CÉPS a réellement été le «C oup du N ord ». Le CÉPS 1999 n'a eu de cesse de prôner l'inclusion et à cet égard, le comité planificateur et ses sous-comités ont toujours su démontrer une grande ouverture d'esprit et une tolérance exceptionnelle aux autres cultures. C ela se reflète dans la volonté de développer un logo identifiant le CÉPS, édition $\mathrm{N}$ ord 
ontarienne 1999. Ce symbole a donné le ton à l'ensemble de l'événement. L'universalité et la simplicité de son dessin interpellent les différentes cultures et permettent différentes interprétations toutes aussi personnelles les unes que les autres. $D$ ans le logo, les couleurs du cercle extérieur représentent les quatre directions, les quatre couleurs de la grande famille humaine et les quatre aspects de l'être - physique, mental, émotif et spirituel. Les quatre volutes intérieures symbolisent le mouvement et représentent les quatre éléments assurant la santé de l'être humain - la terre, l'eau, l'air et le feu. Le cercle du milieu, le noyau, illustre l'essence de l'être, l'énergie, la créativité, en somme, les principes vitaux de chaque individu.

L'inclusion de la composante autochtone et surtout le partage entre les trois composantes culturelles ont été le fait marquant de cette célébration de la promotion de la santé, ce qui en a fait un événement à la fois collectif et individuel. Les participantes et les participants au CÉPS 1999 formaient en quelque sorte un microcosme de la population ontarienne avec les trois cultures fondatrices de notre pays. Par ailleurs, ces valeurs d'inclusion et de partage sont aussi au fondement de la philosophie de prévention et d'un style de vie sain partagée par les promoteurs de la santé et les pourvoyeurs de services de santé.

Le curriculum du CÉPS, édition 1999, était axé sur les rapports entre la personne et sa communauté. C es deux éléments doivent aller de pair. C es rapports comprennent la culture et la spiritualité et prônent l'équilibre, la coopération, le respect et I'innovation. Plusieurs activités du CÉPS comme les visites communautaires, la participation des politiciens municipaux, la présence de médias locaux et la foire de santé avec ses exposants provenant des quatre coins de la province ont permisl'expression de la participation communautaire avec sa diversité culturelle et spirituelle. Le CÉPS s'est même ouvert aux autres communautés provinciales par le biais de la vidéoconférence. Enfin, la technologie et la créativité, comme outils innovateurs en promotion de la santé, complètent la liste des nouveautés de l'expérience $\mathrm{N}$ ord ontarienne du CÉPS. 


\section{Évaluation sommaire et orientations futures}

U ne évaluation du CÉPS, édition 1999, et de ses trois volets sera effectuée, mais déjà il est possible de questionner certains aspects de cet événement. Je soulèverai ici les aspects qui peuvent avoir touché davantage les francophones. II va sans dire que l'ajout de la composante autochtone lui a conféré un statut particulier qui, tout compte fait, constitue un pas en avant dans l'évolution du CÉPS. A près tout, la culture autochtone a intégré, depuis longtemps, une approche globale de promotion de la santé. C ela dit, dans l'édition 1999, les francophones et les autochtones ont dû se partager la moitié de la tarte, l'autre moitié étant accaparée par le volet anglophone.

D èslors, le respect de l'authenticité et de la spécificité de chaque groupe culturel et celui de l'intégration des trois composantes culturelles pour former un tout cohérent posent toujours un problème. Le succès d'une telle entreprise n'est jamais assuré. Si l'ouverture du comité planificateur a permisun partage descultures, il n'en demeure pas moins que la participation des personnes unilingues anglophones à toutes les composantes fut limitée. À I'opposé, en raison de leur bilinguisme, les francophones avaient généralement accès aux meilleurs des trois mondes, puisqu'ils pouvaient choisir parmi les six ateliers parallèles. $C$ et avantage devient vite cependant un casse-tête pour les organisateurs et les animatrices ou les animateurs de sessions en français. La volatilité des participants francophones a certes contribué au faible taux de participation à leur propre atelier. C ela dit, il y avait au moins 28 francophones au CÉPS puisque, lors des sessions parallèles, c'est le nombre qui a été enregistré lors des sessions présentées en français. D onc, l'intérêt de vivre un apprentissage dans sa langue maternelle est toujours bien présent chez lesfrancophones ainsi que la volonté de répondre aux besoins de son groupe d'appartenance.

Par ailleurs, le partage entre les autochtones et les anglophones est plus facile parce qu'ils ont souvent la même langue de communication. D onc, question de rectitude politique, il faudrait 
avoir un service de traduction simultanée pour répondre, en principe, aux besoins des francophones. Je dis bien en principe, car on s'aperçoit très vite qu'un tel service répond davantage aux besoins des anglophones, et non à celui des francophones, afin qu'ils puissent suivre les sessions qui se déroulent en français. C 'est dans cette perspective, me semble-t-il, qu'il faudrait évaluer l'utilité d'un service de traduction simultanée.

Q uoi qu'il en soit, le comité planificateur continuera d'innover dans l'organisation, la structure et les thèmes des CÉPS futurs, peu importe le nombre de composantes culturelles. Toutefois, on doit accorder une attention particulière à la promotion du CÉPS auprès de la clientèle francophone et au volet francophone de cet événement.

En terminant cette évaluation sommaire, j'offrirai quelques pistes de réflexions propres à favoriser le développement de cet événement annuel qu'est le CÉPS. Premièrement, il faut poursuivre et développer davantage le caractère culturel instauré dans l'édition nord-ontarienne du CÉPS 1999. À cette fin, il faut encourager les animatrices et les animateurs à s'exprimer dans leur langue et à utiliser plus souvent le service de traduction simultanée pour faciliter les échanges entre les participants. D euxièmement, il faut continuer à maintenir l'équilibre entre les diverses dimensions de la promotion de la santé et faire du CÉPS, une expérience qui touche les quatre aspects de la personne: mental, physique, spirituel et émotionnel.Troisièmement, il faut explorer la possibilité de publier, sousla forme d'actes de colloques, les présentations faites au CÉPS afin de transmettre les connaissanceset propager le message de l'importance de la promotion de la santé tout au long de l'année. Q uatrièmement, il faudrait axer nos efforts à faire du CÉPS un cours accrédité auprès des diverses instances académiques, dans le but d'accroître la participation. Enfin, l'édition 1999 démontre l'importance de maintenir le caractère itinérant du CÉPS afin d'en faire bénéficier les quatre coins de la province. Pour ce faire, il faudra prévoir des ressources financières qui tiennent compte de la distance, des coûts de transport, de la disponibilité des locaux et des services, en somme, toutes les réalités liées au choix de l'emplacement. 


\section{Conclusion}

Le CÉPS continuera dans le nouveau millénaire à être un outil d'apprentissage pour ceux et celles qui s'intéressent à la promotion de la santé. Selon les évaluations des participantes et participants, il s'agit d'une activité à fortement recommander.

$D$ ans l'avenir, les individus choisiront la santé comme faisant partie intégrante de leur vie. Pour ceux et celles qui choisissent de le faire en français, il faudra veiller à constituer, dans le cadre du CÉPS, un volet francophone fort dans les années à venir. Pour nous en convaincre, rappelons en terminant les propos de $M$ auril Bélanger, secrétaire parlementaire de Patrimoine canadien, qui soulignait l'importance «de former des professionnels de la santé en français pour assurer à la population francophone des soins de santé dans sa langue ».

\section{Bibliographie}

DER O CHE, Francine (1999, 3 juin). Synthèse du C ours d'été en promotion de la santé de l'O ntario à Sudbury, Sudbury.

U N IVER SITÉ D'OTTAWA (1999). «C ampus, le Centre francophone national de formation en santé », M agaz ineTabaret, vol. 48, no 3, 21. 